\title{
DNA methylation and gene expression
} changes in mouse pre- and post-implantation embryos generated by intracytoplasmic sperm injection with artificial oocyte activation

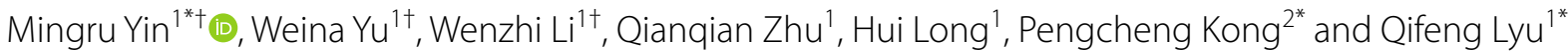

\begin{abstract}
Background: The application of artificial oocyte activation (AOA) after intracytoplasmic sperm injection (ICSI) is successful in mitigating fertilization failure problems in assisted reproductive technology (ART). Nevertheless, there is no relevant study to investigate whether $\mathrm{AOA}$ procedures increase developmental risk by disturbing subsequent gene expression at different embryonic development stages.
\end{abstract}

Methods: We used a mouse model to explore the influence of AOA treatment on pre- and post-implantation events. Firstly, the developmental potential of embryos with or without AOA treatment were assessed by the rates of fertilization and blastocyst formation. Secondly, transcriptome high-throughput sequencing was performed among the three groups (ICSI, ICSI-AOA and dICSI-AOA groups). The hierarchical clustering and Principal Component Analysis (PCA) analysis were used. Subsequently, Igf2r/Airn methylation analysis were detected using methylation-specific PCR sequencing following bisulfite treatment. Finally, birth rate and birth weight were examined following mouse embryo transfer.

Results: The rates of fertilization and blastocyst formation were significantly lower in oocyte activation-deficient sperm injection group (dICSI group) when compared with the ICSI group (30.8\% vs. 84.4\%, $10.0 \%$ vs. $41.5 \%$ ). There were 133 differentially expressed genes (DEGs) between the ICSI-AOA group and ICSI group, and 266 DEGs between the dICSI-AOA group and ICSI group. In addition, the imprinted gene, Igf2r is up regulated in AOA treatment group compared to control group. The Igf2r/Airn imprinted expression model demonstrates that AOA treatment stimulates maternal allele-specific mehtylation spreads at differentially methylated region 2 , followed by the initiation of paternal imprinted Airn long non-coding (Inc) RNA, resulting in the up regulated expression of Igf2r. Furthermore, the birth weight of newborn mice originating from AOA group was significantly lower compared to that of ICSI group. The pups born following AOA treatment did not show any other abnormalities during early development. All offspring mated successfully with fertile controls.

\footnotetext{
*Correspondence: 315021@sh9hospital.org.cn; kongpengcheng@51mch. com; LVQF3327@sh9hospital.org.cn

${ }^{\dagger}$ Mingru Yin, Weina Yu and Wenzhi Li contributed equally to this work.

${ }^{1}$ Department of Assisted Reproduction, Shanghai Ninth People's Hospital,

Shanghai Jiao Tong University School of Medicine, 200011 Shanghai,

China

2 Department of Assisted Reproduction, First Maternity and Infant

Hospital, Tongji University School of Medicine, 201204 Shanghai, China
}

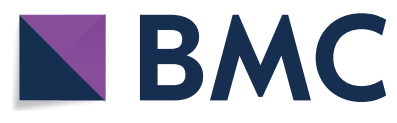

(c) The Author(s) 2021. Open Access This article is licensed under a Creative Commons Attribution 4.0 International License, which permits use, sharing, adaptation, distribution and reproduction in any medium or format, as long as you give appropriate credit to the original author(s) and the source, provide a link to the Creative Commons licence, and indicate if changes were made. The images or other third party material in this article are included in the article's Creative Commons licence, unless indicated otherwise in a credit line to the material. If material is not included in the article's Creative Commons licence and your intended use is not permitted by statutory regulation or exceeds the permitted use, you will need to obtain permission directly from the copyright holder. To view a copy of this licence, visit http://creativecommons.org/licenses/by/4.0/. The Creative Commons Public Domain Dedication waiver (http://creativeco mmons.org/publicdomain/zero/1.0/) applies to the data made available in this article, unless otherwise stated in a credit line to the data. 
Conclusions: AOA treatment affects imprinted gene lgf2r expression and mehtylation states in mouse pre- and postimplantation embryo, which is regulated by the imprinted Airn. Nevertheless, no significant differences were found in post-natal growth of the pups in the present study. It is hoped that this study could provide valuable insights of AOA technology in assisted reproduction biology.

Keywords: Artificial oocyte activation, Mouse blastocysts, Gene expression, Imprinted gene, DNA methylation

\section{Introduction}

Assisted reproductive technology (ART) provide a useful alternative for the infertility cases and five million children were born to date using these methods. The application of intracytoplasmic sperm injection (ICSI) has solved most male infertility problems in assisted reproductive medicine [1]. Whereas, $1-3 \%$ of ICSI fertilization failure still occurs in ICSI cycles [2]. Oocyte activation deficiency (AOD) is one of the factors associated with ICSI failure. Oocyte activation is an imperative stage in the initiation of embryo development during the fertilization, involving mainly calcium $\left(\mathrm{Ca}^{2+}\right)$ rise. The calcium oscillations are stimulated by the entrance of sperm, and then phosphatidylinositol 4,5-bisphosphate (PIP2) is hydrolyzed to inositol 1,4,5-trisphosphate (IP3) and diacylglycerol (DAG) by some sperm soluble factors. Whereafter, further $\mathrm{Ca}^{2+}$ oscillations are induced following IP3 binds to specific receptors. A series of molecular events such as exocytosis of cortical granules, recruitment of maternal mRNA, pronuclear formation, and polyspermy prevention occurred after oocyte activation [3-5]. Phospholipase C zeta (PLCל) existed in sperm, which is considered to be the physiological factor for oocyte activation [6]. Apart from these natural activators, some stimulants, such as ionomycin, calcimycin, and strontium chloride have been applied to mitigate ICSI fertilization failure. These methods have been known as artificial oocyte activation (AOA) and are considered to be a useful method for fertilization failure problems.

With the increasingly wide application of AOA, some concerns remain regarding the safety of this technology. The period that oocyte activation is a critical window of gene imprinting removal, establishment and maintenance. So it is critically important to investigate whether AOA procedure increase developmental risks during the embryonic or neonatal development stages. Although some clinical data analyses have demonstrated that birth characteristics and congenital malformations following AOA are within the expected range [7-9], it is critically important to evaluate in details the impact of AOA in embryo early development from gene expression and epigenetic point of view. Therefore, it is essential to animal models for further study. Mouse oocytes can be used to study oocyte activation in humans [10]. Therefore, a mouse model with oocyte AOD sperm would be useful for studying AOA. Some sperm-derived factors, such as PLC $\zeta$ [11], are considered as the sperm-associated oocyte-activating factors (SOAFs). Some studies $[12,13]$ have proved that release of SOAFs from disrupted membranes had been shown to suppress oocyte activation. In our previous study, we established a simple and effective method for generating oocyte AOD mouse spermatozoa [14]. And with the release of SOAFs from disrupted membranes, spermatozoa with different oocyte activation-deficient were established.

Precise genomic imprinting plays an important role in regulating the growth and development of embryos, and also in the early postnatal phase. Manipulation or nonphysiological culture environments may affect the gene expression or epigenetic modification of imprinted genes during early embryo development stages or later fetal development. DNA methylation, an epigenetic imprinting modification, acts in concert to negatively control gene expression. DNA methylation at differentially methylated regions (DMRs) or imprinting control regions (ICRs) is required for imprinted expression at many loci. But until now, it is unknown whether AOA treatment would produce some effects on gene expression and methylation in embryo development stages. Therefore, we aim to explore these in this study.

In the present study, transcriptome high-throughput sequencing was performed to provide a profile of the differences in the global patterns of gene expression between ICSI-AOA treatment and ICSI generated mouse blastocysts. In addition, we focused on the different expression of imprinted genes, of which the Igf $2 r$ was up-regulated in AOA treatment groups compared to ICSI group. It is known that the imprinted Airn long noncoding (lnc) RNA is paternally expressed and silences Igf $2 r$ genes in cis. Therefore, the study aims to explore the effect of AOA on the dynamic regulation of Igf2r/Airn in the pre- and post-implantation embryo.

\section{Materials and methods \\ Animals}

C57BL/6 mice were provided by the SLAC Laboratory Animal Co. Ltd (Shanghai, PRC) and were used to prepare oocytes and sperms. All animals were maintained under a 12:12 h lighting schedule rooms (lights on at 07:00-19:00). All animals were maintained and treated in 
accordance with the National Institutes of Health Guide for the Care and Use of Laboratory Animals and were approved by Animal Care and Use Committee of Shanghai Jiao Tong University School of Medicine.

\section{Mouse oocyte collection and activation-deficient spermatozoa handling}

Mouse oocyte collection process was shown in our previous study $[14,15]$. Female C57BL/6 mice (4-6 weeks old) were used for superovulation. Finally, the denuded oocytes were cultured in drops of KSOM (MR-107, Millipore, Germany) under mineral oil at $37{ }^{\circ} \mathrm{C}$ and $5 \%$ $\mathrm{CO}_{2}$ in the incubator. An efficient method for producing activation-deficient mouse spermatozoa was identified in previous study [14]. Briefly, Epididymal spermatozoa were obtained from male $\mathrm{C} 57 \mathrm{BL} / 6$ mice at 8-12 weeks. A dense sperm mass was incubated at $37^{\circ} \mathrm{C}$ and $5 \% \mathrm{CO}_{2}$ for 30 min to gather motile sperm. A half of the top $1 \mathrm{ml}$ sperm suspension was carefully aspirated. Then sperm suspension was rapidly drawn in and out by a $1 \mathrm{ml}$ pipette for $10 \mathrm{~min}$ to separate $60-80 \%$ of sperm heads from their tails. To acquire oocyte-activating capacity decreased sperm, the sperm heads were incubated at $37^{\circ} \mathrm{C}$ for $4 \mathrm{~h}$. The other half of the top $1 \mathrm{ml}$ sperm suspension was directly injected into oocytes for the control.

\section{Measurement of calcium oscillation pattern}

Firstly, MII mouse oocytes were loaded in KSOM with $10 \mu \mathrm{M}$ fura-2 acetoxymethyl ester (fura-2 AM, Invitrogen) for $20 \mathrm{~min}$ at $37{ }^{\circ} \mathrm{C}$. The dye was first dissolved in DMSO containing Pluronic F-127 (Molecular Probes no. P3000) (final concentration, $0.08 \% \mathrm{v} / \mathrm{v}$ and $0.016 \%$, respectively) and then diluted in KSOM-HEPES medium. After washing three times, the loaded oocytes were incubated in KSOM medium at $37{ }^{\circ} \mathrm{C}$ under $5 \% \mathrm{CO}_{2}$. Then the oocytes were used for ICSI or ICSI-AOA in different groups under the conditions described. And the oocytes were allowed at least 20 min of recovery in KSOM. Next, the oocytes were placed in drops of KSOM under mineral oil on an inverted microscope equipped with an atmospheric chamber and a filter switch providing excitation alternating between 340 and $380 \mathrm{~nm}$ for fura2 , and the emission wavelength is $510 \mathrm{~nm}$. On average, 10-15 oocytes in each group were measured simultaneously. A scan was performed every $5 \mathrm{~s}$ over a time period of $1 \mathrm{~h}$. The intracellular calcium levels were recorded in terms of the ratio of 340 and $380 \mathrm{~nm}$ fluorescence. Four different categories were distinguished according to the frequency of calcium oscillatory pattern as follows [16, 17]with appropriate changes: $(0)$ no $\mathrm{Ca}^{2+}$ spikes, $(+)$ $1-3$ spikes, $(++) 4-8$ spikes and $(+++)>8$ spikes within $1 \mathrm{~h}$ following ICSI.

\section{Mouse ICSI and artificial oocyte activation (AOA)}

The microinjection was performed on an inverted microscope (Olympus) equipped with a warm plate and a micromanipulation system (MM-89; Narishige, Iapan) as previously described [18]. The oocytes were treated with ionomycin (Sigma, St. Louis, MO, USA) in different concentrations for $10 \mathrm{~min}$ at $37{ }^{\circ} \mathrm{C}$ in $5 \% \mathrm{CO} 2$, after $30 \mathrm{~min}$ of ICSI. Our study contained 4 groups, as follows: (A, ICSI group) normal sperm injection as control, (B, ICSI-AOA group) normal sperm injection with AOA treatment, (C, dICSI group) oocyte activation-deficient sperm injection, (D, dICSI-AOA group) oocyte activation-deficient sperm injection with AOA treatment. To determine the optimal concentration for each treatment, the dICSI-derived oocytes were treated with concentrations of 0 (solvent control), $1 \mu \mathrm{M}, 2.5 \mu \mathrm{M}$ ionomycin for $10 \mathrm{~min}$. The suitable AOA condition was determined by calcium oscillation pattern analysis compared with group A. Then the oocytes were thoroughly washed and left in KSOM for further culture or for the next calcium oscillation pattern analysis.

\section{Embryo transfer}

Female ICR mice aged 8-12 weeks old were used as recipient mothers. The night before embryo transfer, they were mated with vasectomized ICR male mice to obtain pseudopregnant foster mothers. According to the presence of a vaginal plug, surrogate ICR mice were identified, and the day of the vaginal plug was designated as 0.5 d.p.c. (days post coitum). Each time, 14-18 embryos were transferred at the 2-cell stage into the oviduct of each recipient using a glass pipette. The days of fetal sampling were 6.5 d.p.c., 7.5 d.p.c., and 8 d.p.c., respectively. After birth, pups were weighed weekly until 8 weeks of age. Pups were weaned after the third week, and females and males were put separately. At 8 weeks of age, couples were formed by putting male and female offspring with fertile controls to assess their fertility.

\section{Collection of Samples and Transcriptome High-Throughput Sequencing}

The blastocyst stage embryos were collected for sequencing. Five blastocysts were pooled for each treatment group (ICSI, ICSI-AOA and dICSI-AOA) in the same time from started injection and three independent replicates for each treatment procedure were collected. ICSI, ICSI-AOA and dICSI-AOA were named group A, $B$ and $D$, respectively in the following sequencing. Total RNA was isolated from blastocyst of samples with TRIzol reagent (Life Technologies, Carlsbad, CA, USA) and GlycoBlue $^{\mathrm{TM}}$ Coprecipitant (Ambion, AM9515) for facilitating good RNA recovery while increasing the size and 
visibility of the pellet. The RNA concentration of each sample was measured using the NanoDrop ND-1000 instrument (Thermo Fisher Scientific, Waltham, MA, USA). All RNA samples met standards of quality control according to the qualified ratio of OD260/OD280 ranged from 1.8 to 2.1. Transcriptome high-throughput sequencing was provided by CloudSeq Biotech Inc. (Shanghai, China). RNA preprocessing for constructing the sequencing library was implemented with the SMARTer Stranded Total RNA-Seq Kit (Takara). Libraries were controlled for quality and quantified using the BioAnalyzer 2100 system (Agilent Technologies, Santa Clara, CA, USA).

\section{Bioinformatics analysis}

Paired-end reads were acquired from the Illumina HiSeq 4000 sequencer. After $3^{\prime}$ adaptor-trimming and removing low quality reads by cut adapt software (v1.9.3), the high quality trimmed reads were used to analyse mRNAs. The high quality reads were aligned to the mouse reference genome (UCSC mm10) with hisat2 software. Then, cuff diff software (part of cufflinks) was used to get the FPKM as the expression profiles of mRNA guided by the Ensembl gtf gene annotation file, and fold change and p-value were calculated according to FPKM, differentially expressed mRNA were identified. Alternative splicing was performed by SplAdder software. The statistical significance differentially expressed mRNAs (fold changes $>1.3$ and $p<0.05$ ) were identified. The data had been deposited in the National Center for Biotechnology Information (NCBI) Gene Expression Omnibus (GEO). The GEO accession number is GSE155948.

\section{RNA extraction and Q-PCR}

Each group of blastocyst pool was separately collected and performed according to the Cell Amp Whole Transcriptome Amplification Kit (TaKaRa, Japan). cDNA was amplified through 20 cycles of PCR. Subsequently, 10 -fold diluted cDNA was used as the template for Q-PCR, which was performed based on the SYBR Green I Real-Time PCR Kit (TaKaRa). The primers were presented in Supplementary Table S1. Each gene was tested in triplicate.

\section{DNA bisulfite treatment}

Bisulfite mutagenesis was performed as previously described [15], with one blastocyst in each pool. Briefly, the DNA was wrapped in agarose gels to form little beads. The beads were treated with sodium bisulfite based on an EZ DNA Methylation Kit (Zymo Research, Orange, CA). The beads were immediately used for PCR or stored at $-20{ }^{\circ} \mathrm{C}$. Methylation-specific PCR (MS$\mathrm{PCR}$ ) primers were used to match the allele-specific
DNA strands (Supplementary Table S1). Following nested PCR, cloning and sequencing were performed as previously described [15]. Briefly, the first round of PCR was conducted using one bead containing bisulfitetreated DNA with the outer primers and Taq HS premix (TaKaRa). For the second round of PCR, MS-PCR primers and $2 \mu \mathrm{l}$ of the first PCR amplified products were used. To identify the specific amplification in PCR, $5 \mu \mathrm{l}$ of each second round PCR product was separated by agarose gel electrophoresis. Next, the remaining PCR amplified products were cloned into a T vector (PMDTM19-T Vector Cloning Kit, TaKaRa) and individual clones were sequenced. The bisulfite sequencing PCR was also carried out according to the above protocols.

\section{Statistical analysis}

All analyses were performed using SPSS, version 21.0 software (SPSS Inc., Chicago, IL, USA). Quantitative data are expressed as mean \pm standard deviation (SD). Comparisons between groups were performed with $t$ test or Chi-squared test. Principle component analysis was performed using ADE4 package of $\mathrm{R}$ according to the standardized expression profile data. Values of $P<0.05$ were considered to be significantly different.

\section{Results}

\section{Calcium oscillation pattern analysis}

Firstly a standard calcium oscillatory profile was evaluated in the mouse oocytes with injection of normal sperm. The frequency of calcium oscillations was scored per oocytes within $1 \mathrm{~h}$ following ICSI (Table S2). Representative traces were shown in Fig. 1 A. We defined $\geq 1 \mathrm{Ca}^{2+}$ spike/recording period triggered as 'oscillatory activity'. Calcium oscillation analysis following ICSI with oocyte activation-deficient sperm (dICSI) demonstrated that $33.33 \%$ of the injected mouse oocytes showed oscillatory activity, whereas $93.33 \%$ of the injected oocytes using normal sperm showed a highly calcium rises $(P<0.05$, Table S2). The average number of calcium oscillatory per responding oocytes injected with oocyte activation-deficient sperm was significantly lower (3.56 rises, range 1-8) than that of injected oocytes using normal sperm (9.52 rises, range 1-16) $(P<0.05$, Table S1). Amplitude of calcium rises had no significant difference. No calcium rises were observed following the non-injected oocytes $(n=10)$ or sham injection oocytes $(n=10)$. We next explored the influence of AOA on the calcium oscillation pattern in mouse oocytes injected with oocyte activation-deficient sperm. $1 \mu \mathrm{M}$ and $2.5 \mu \mathrm{M}$ ionomycin were used for oocyte activation in order to choose the suitable AOA condition by calcium oscillation pattern analysis compared with control ICSI group (Table S3 \& Fig. 1B). Frequency 
A
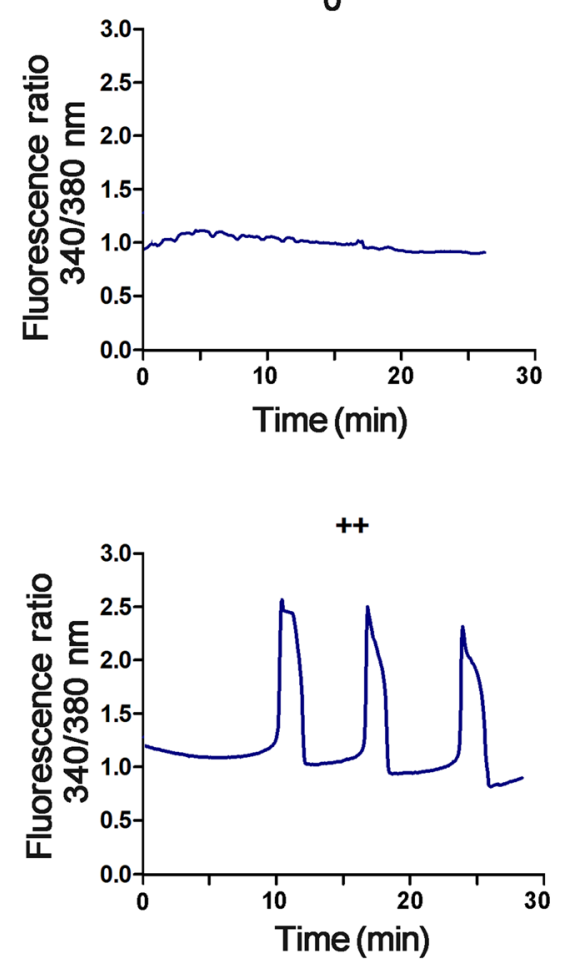
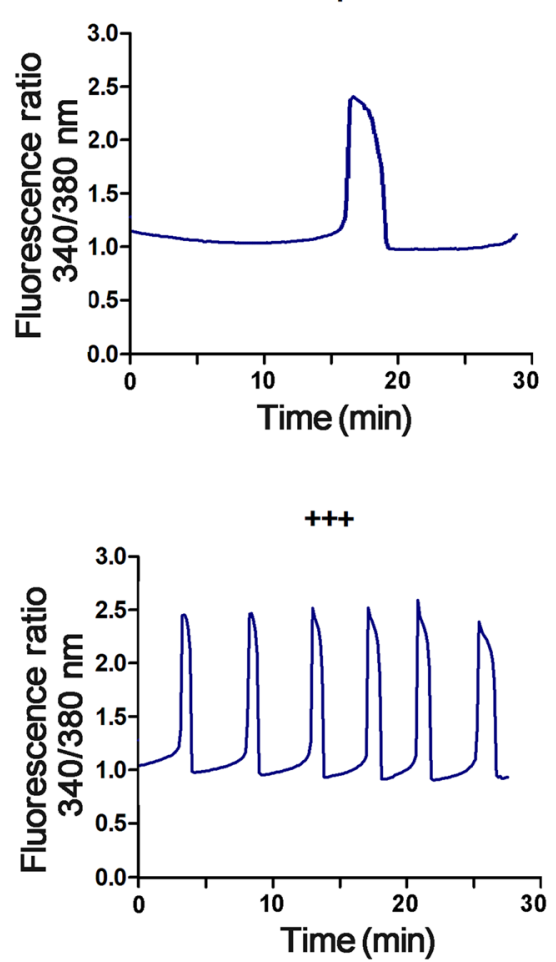

B

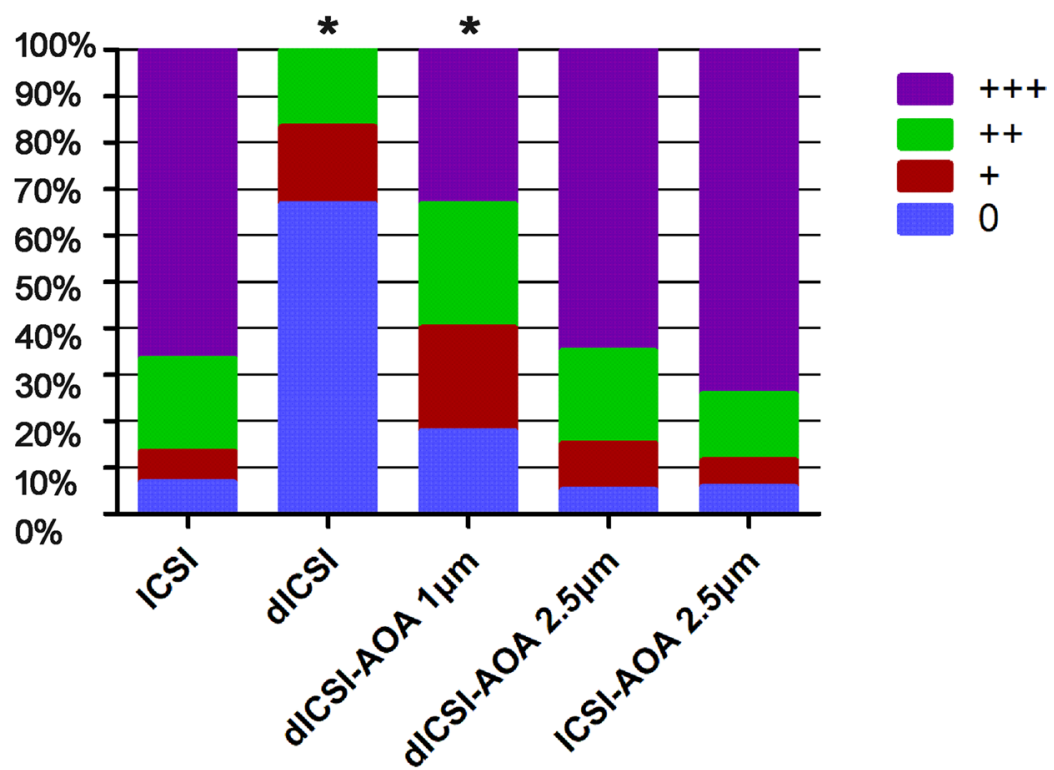

Fig. 1 Calcium oscillation pattern analysis. A Representative calcium oscillation patterns for the calcium frequency scoring following normal mouse spermatozoa. The frequency score per oocyte is given based on the number of calcium rises seen during the $1 \mathrm{~h}$ measuring period: ' $+++{ }^{\prime},>8$ spikes; +++ ', 4-8 spikes; '+', 1-3 spikes; '0', no calcium spikes seen. B Frequency scoring of the calcium oscillations following normal mouse spermatozoa or oocyte activation-deficient mouse spermatozoa ICSI with different concentration of ionomycin. ${ }^{*}$ indicates distribution of frequency pattern significant differences compared to ICSI group, $P<0.05$ 
analysis in dICSI-AOA with different ionomycin concentrations demonstrated that the distribution of frequency patterns in $2.5 \mu \mathrm{M}$ ionomycin was comparable to that of ICSI group. Therefore we considered that at least $2.5 \mu \mathrm{M}$ ionomycin was needed to support the oocyte activation-deficient sperm to generate normal $\mathrm{Ca}^{2+}$ response activity. So $2.5 \mu \mathrm{M}$ ionomycin was used for the following experiment.

\section{Effects of artificial oocyte activation treatment on the embryo development in vitro}

To determine the effect of AOA on the fertilization and early embryonic development of ICSI-derived embryos. AOA was performed following ICSI injected with normal sperm (B, ICSI-AOA group) or oocyte activationdeficient sperm (D, dICSI-AOA group). The normal sperm injection as control (A, ICSI group). The results showed in Table 1 indicated that the rate of fertilization decreased notably in oocyte activation-deficient sperm ICSI group (C, dICSI group) compared to ICSI group

Table 1 Effects of AOA treatment on the development of ICSIderived embryos in vitro

\begin{tabular}{llll}
\hline Group & $\begin{array}{l}\text { No. of } \\
\text { surviving } \\
\text { oocytes }\end{array}$ & $\begin{array}{l}\text { No. of } \\
\text { fertilization } \\
\text { oocytes (\%) }\end{array}$ & $\begin{array}{l}\text { No. of blastocyst } \\
\text { stage oocytes(\%) }\end{array}$ \\
\hline A, ICSI group & 77 & $65(84.4)^{\mathrm{a}}$ & $27(41.5)^{\mathrm{a}}$ \\
B, ICSI-AOA group & 71 & $59(83.1)^{\mathrm{a}}$ & $24(40.7)^{\mathrm{a}}$ \\
C, dICSI group & 65 & $20(30.8)^{\mathrm{b}}$ & $2(10)^{\mathrm{b}}$ \\
D, dICSI-AOA & 72 & $57(79.2)^{\mathrm{a}}$ & $21(36.8)^{\mathrm{a}}$ \\
group & & & \\
No injection + & 18 & 0 & 0 \\
AOA & & & \\
\hline
\end{tabular}

$\mathrm{a}, \mathrm{b}$ With the same column are significantly different $(P<0.05)$
(30.8\% vs. $84.4 \%, P<0.05$ ), which was rescued after AOA treatment. Furthermore, there was no statistically significant difference in the rate of fertilization between ICSIAOA group and ICSI control group $(83.1 \%$ vs. $84.4 \%, P$ $>0.05)$. And $2.5 \mu \mathrm{M}$ ionomycin did not induce parthenogenetic activation of mouse oocytes. In addition, AOA treatment in group $\mathrm{D}$ has the higher blastocyst formation rate than that of dICSI-derived embryos (36.8\% vs. $10 \%$, $P<0.05)$. Moreover, there were no significant differences in the rate of blastocyst formation among group A, group $\mathrm{B}$ and group D $(41.5 \%, 40.7 \%$ and $36.8 \%$, respectively, $P$ $>0.05$ ). The representative images of early embryo development were shown in Fig. 2. Thus, the results revealed that AOA treatment could improve the fertilization and blastocyst formation of oocyte activation-deficient sperm ICSI-derived embryos in vitro. Furthermore, moderate AOA treatment did not affect fertilization and embryo development in normal ICSI group.

\section{Profiles of Differentially Expressed mRNAs}

The blastocyst stage embryos were collected for the sequencing. The sequencing analysis contained 3 groups, as follows: (A, ICSI group) normal sperm injection as control, (B, ICSI-AOA group) normal sperm injection with $2.5 \mu \mathrm{M}$ ionomycin treatment, (D, dICSI-AOA group) oocyte activation-deficient sperm injection with $2.5 \mu \mathrm{M}$ ionomycin treatment. After high-throughput sequencing, the heat map showed the hierarchical clustering of the distinguishable mRNA expression profiling among three groups (Fig. 3 A). Hierarchical clustering analysis using differentially expressed gene set make the samples into two major clustering branches. Three replicate samples in ICSI group self-cluster into one branch and the samples of ICSI-AOA and DICSI-AOA group

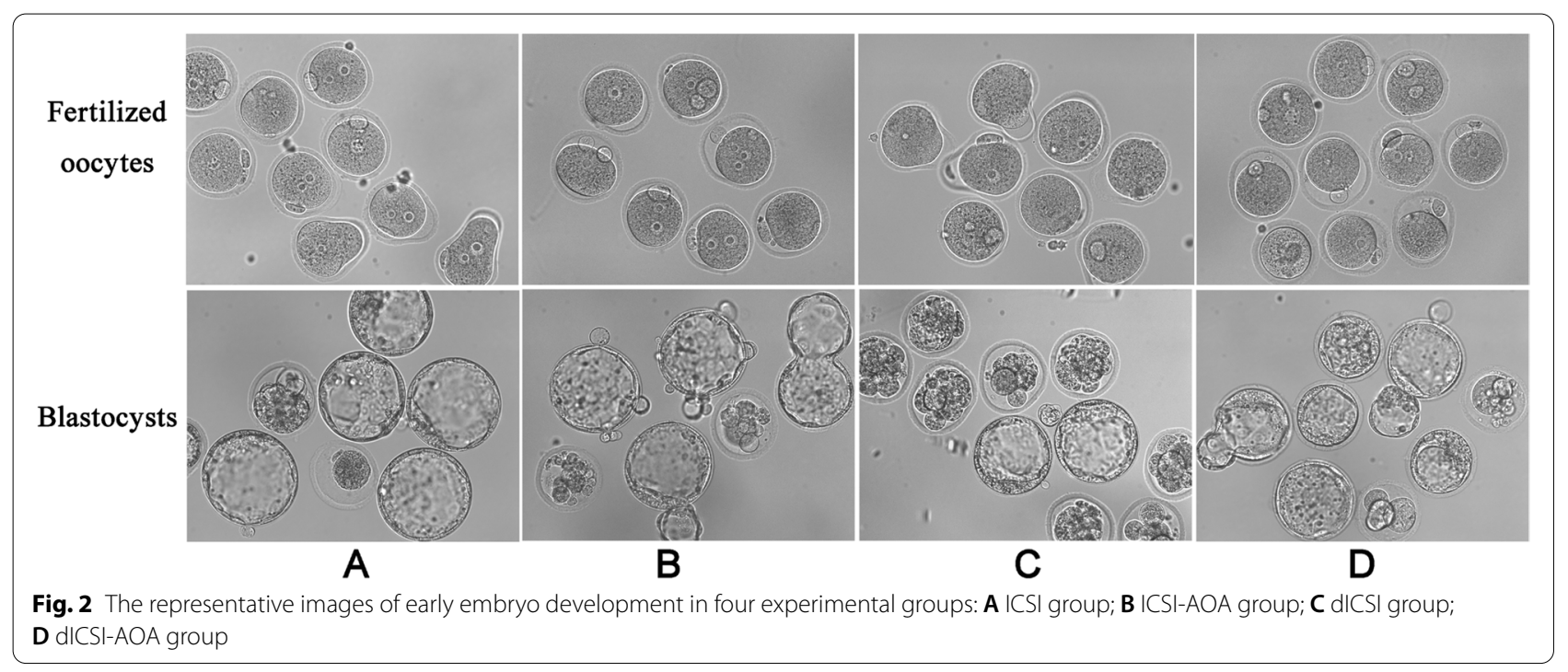




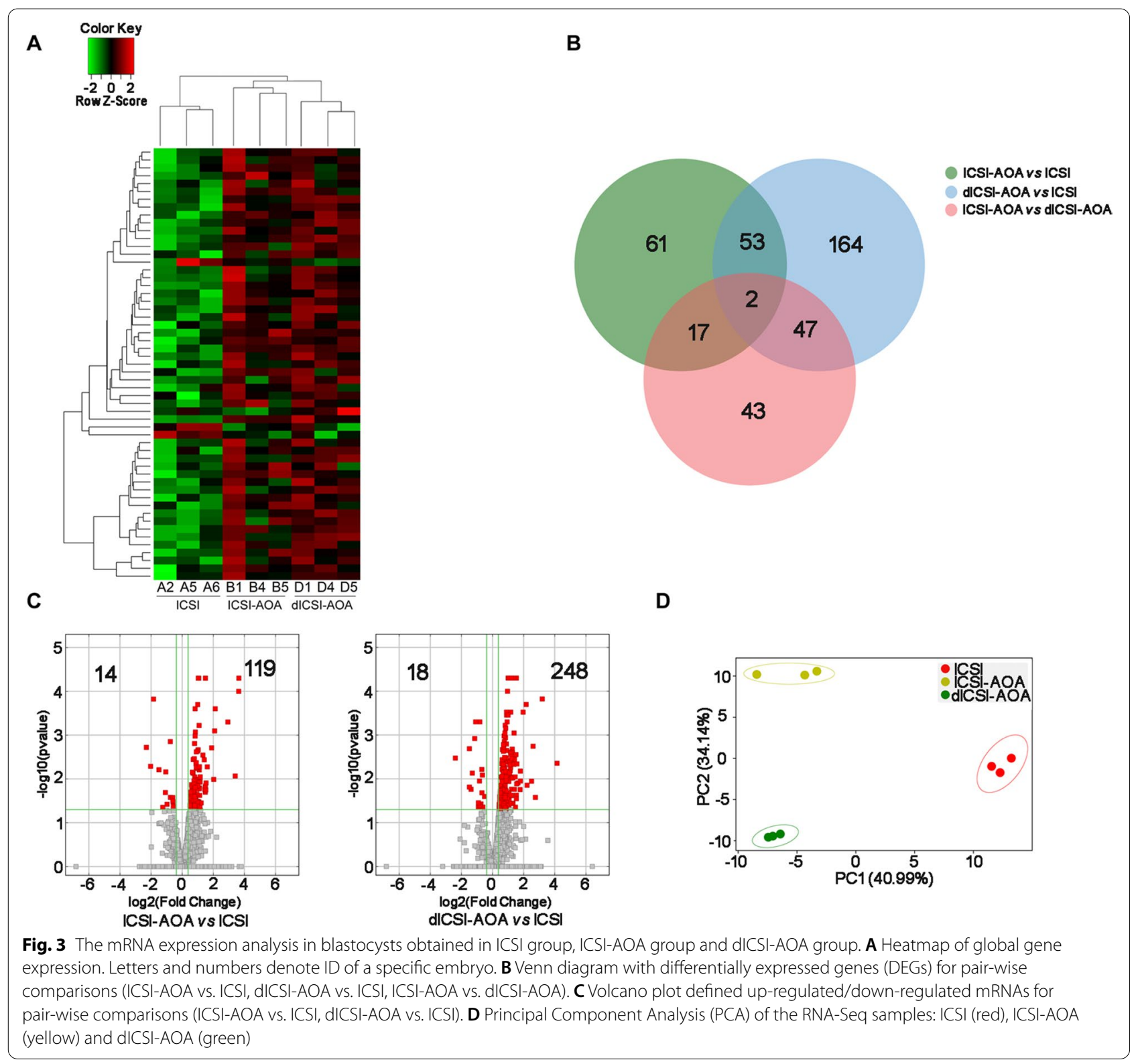

cluster into another branch. Moreover, all the replicates of ICSI-AOA and dICSI-AOA group self-cluster into two major sub-branches. In the process of differential analysis of mRNAs, the results showed that there were 133 differentially expressed genes (DEGs) between the ICSIAOA group and ICSI group, and 266 DEGs between the dICSI-AOA group and ICSI group (Fig. 3B). The DEGs for pair-wise comparisons was listed in table S4. There was a higher number of up-regulated (119/133-89.5\% in ICSI-AOA group and 248/266-93.2 \%in dICSI-AOA group) than down-regulated (14/133-10.5\% and 18/266$6.8 \%$, respectively) in significantly different genes. Each dot stood for an mRNA in the volcano plot (Fig. 3 C). The red dots on the left side stood for the down-regulated mRNAs and those on the right side stood for the upregulated mRNAs with significant differences, while the grey dots stood for the mRNAs which expressed differentially without significance. Principal Component Analysis (PCA) distributed samples into a two dimensional space according to the variance in gene expressions, and samples with similar trends in gene expression profiles will cluster together in the PCA plot. The result showed that 9 samples clearly segregate into three clusters, corresponding to ICSI, ICSI-AOA and dICSI-AOA group (Fig. 3D). PCA analysis demonstrated that the embryos from each 


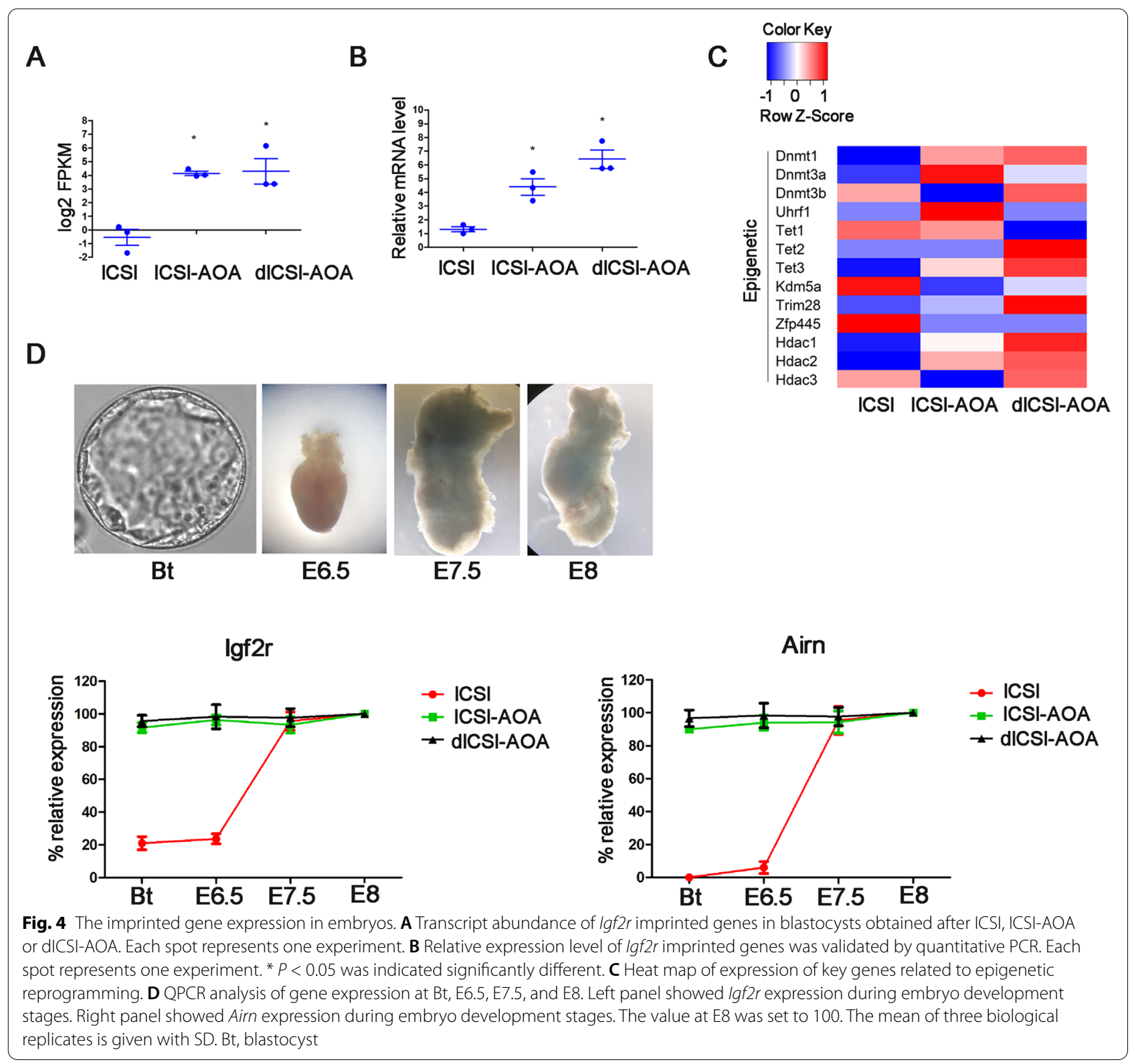

group clustered together, despite expected individual variability.

\section{Expression of imprinted genes in embryos with artificial oocyte activation treatment}

To investigate the epigenetic changes of AOA method in ART technology, we evaluated the effect of AOA treatment on the imprinted genes expression. In the process of differential analysis of imprinted genes, there were two differentially expressed imprinted genes between ICSI-AOA group and ICSI group, and one differentially expressed imprinted gene between dICSI-AOA group and ICSI group. Igf $2 r$ was both up-regulated in
ICSI-AOA group $(\mathrm{FC}=22.786)$ and dICSI-AOA group $(\mathrm{FC}=38.849)$ compared to that of ICSI group. The expressions of $I g f 2 r$ in three groups were validated by quantitative PCR, which was consistent with the sequencing results (Fig. 4 A\&B). Moreover, in order to evaluate the effect of AOA manipulation on the expression of genes associated with epigenetic reprogramming, we analyzed the expression of key genes related to methylation, demethylation and remethylation process. The result was presented in the heat map in Fig. 4 C. Meanwhile, we compared these genes in DEGs. Despite of some genes with a wide range in transcript abundance, there were no 
significant differences in these gene expressions for pairwise comparisons.

\section{Methylation states of Igf $\mathbf{r}$ in blastocysts derived from artificial oocyte activation treatment}

The Igf2r locus has two known DMRs (Fig. 5 A). The methylation profiles of the imprinted genes Igf2r promoter (DMR1) were analyzed in blastocysts obtained from three experimental groups. The number of $12 \mathrm{CpG}$ sites were examined in the $I g f 2 r$ promoter. The results were presented in a 'lollipop' format (Fig. 5B). The sequencing results were derived from unmethylatedspecific primers. When we used methylated-specific primers, no PCR products were obtained. We concluded that DMR1 of Igf2r showed unmethylated status on both parental alleles in these three groups (Fig. 5B). The results suggest that AOA treatment does not affect DMR1 DNA mehtylation. From another point of view, it can be explained that DNA mehtylation in DMR1 does not regulate the expression of $I g f 2 r$ in preimplantation embryo.

\section{Dynamic regulation of Igf2r/Airn in the pre-implantation embryo}

Figure S1 depicts the previous known of Igf2r/Airn imprinted expression model. In preimplantation embryos or undifferentiated ES cells, Igf2r is expressed biallelically and Igf $2 r$ promoter is unmethylated, Airn is silent but the maternal Airn promoter carries a DNA methylation imprint. In late post-implantation or late differentiated ES cells, Igf2r is up-regulated on the maternal allele, but the up-regulation is blocked by Airn transcriptional interference in cis on the paternal allele. The paternal Igf $2 r$ maintains low-level expression and gains DNA methylation in late post-implantation or late differentiation. Since Airn is considered to regulate the expression of $I g f 2 r$, we examined the relative mRNA expression levels of Airn in three groups at blastocyst stage. The relative expression results were shown in Fig. 4D. Airn was expressed in AOA-derived blastocyst. However, no Airn was detected in control ICSI-derived blastocyst. These results indicate that AOA treatment might initiate Airn transcription to regulate the $I g f 2 r$ expression.

To further explore the methylated regulation of $I g f 2 r /$ Airn, we next analyzed the methylation in DMR2. DMR2 methylation has been proved to be present in oocytes. Previous reports demonstrated DMR2 present the precise 3' boundary of ICR mehtylation [CpG at Chr17:12,742,488-12,742,489] in oocytes. Next, the methylation profiles of DMR2 including 3'boundary was analyzed in oocytes. We examined $13 \mathrm{CpG}$ sites in DMR2. The results showed that the precise ICR border was maintained in oocytes (Fig. 5C). Next, the methylation profiles of DMR2 including 3' boundary was analyzed in blastocysts obtained from three experimental groups. In ICSI control blastocysts, the precise 3' ICR border was detected in some clones. However, DNA methylation showed spreading in the 3'direction in AOA treatment blastocysts (Fig. 5D). These results indicated that methylation at DMR2 may increase or spread in cells of preimplantation embryo with AOA treatment. In order to better explore the methylation of specific allele, the methylation-specific PCR (MS-PCR) primers in two flanks of 3 ' boundary were designed for DMR2 methylation analysis in three experiment groups (Table S1). We examined $10 \mathrm{CpG}$ sites and $7 \mathrm{CpG}$ sites in DMR2, respectively (Figure S2). For the left side of 3' boundary, the maternal allele was methylated allele, the paternal allele was unmethylated allele. For the right side of 3'boundary, the DNA strand containing the methylated allele was not detected using methylated-specific primers in ICSI group. In other words, the precise 3' border was maintained on the maternal allele in ICSI control blastocysts. However, DNA methylation had spread in the 3' direction in AOA treatment blastocysts. These results indicated that maternal allelespecific methylation at DMR2 increases or spreads in pre-implantation embryo with AOA treatment.

In our model, Fig. 5E showed a summary schematic depiction about regulation of Igf2r/Airn in blastocyst embryos obtained from three groups. In ICSI control blastocysts, $I g f 2 r$ is expressed biallelically and DMR1 is unmethylated on both parental alleles. Airn is silent and the maternal DMR2 carries a DNA methylation imprint region in which the precise ICR border mehtylation was maintained. In AOA treatment ICSI blastocysts, maternal DMR2 methylation spreads, followed by the initiation of paternal Airn transcription. Igf2 $r$ is up regulated expression on the maternal allele, but the up-regulation is blocked by Airn transcriptional interference in cis on the paternal allele. The paternal Igf2r maintains low-level expression. It is showed that the extends of DMR2 methylation is coincident with initiation of Airn expression in AOA treatment blastocysts, demonstrating that AOA treatment stimulates maternal allele-specific mehtylation spreads at DMR2, followed by initiation of paternal Airn transcription, resulting up regulated expression of $I g f 2 r$.

\section{Expression of Igf2r/Airn in the post-implantation embryo}

To further explore the expression of Igf2r/Airn in the post-implantation embryo with AOA treatment, we transferred ICSI, ICSI-AOA and dICSI-AOA embryos into surrogate mothers. Next, we used QPCR to quantify the expression of Igf2r and Airn at E6.5, E7.5, and E8. Figure 4D showed that Igf2r and Airn expression 


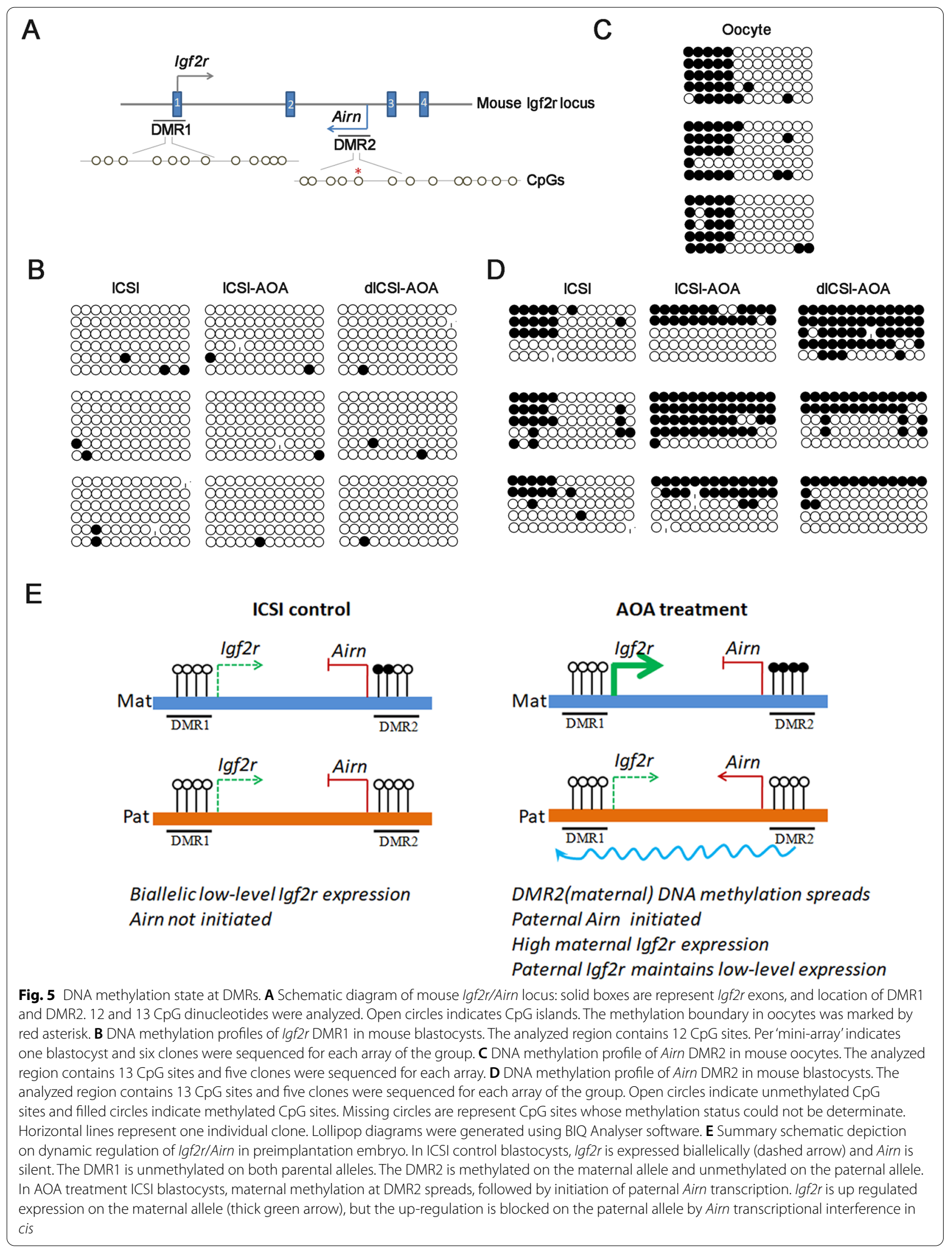


increased during embryo development stages: a sharp increase was observed between E6.5 and E7.5 for both genes in ICSI control group. Relative to blastocysts, Igf $2 r$ increased on average $\sim 5$-fold and Airn increased $\sim 100$-fold in ICSI control group. However, the expression of Igf2r and Airn did not change in AOA treatment group during embryo development stages. Relative to ICSI control blastocysts, the gene expression in AOA treatment embryo at blastocyst and E6.5 was the same as that of ICSI control embryo at E7.5. These results further demonstrated that AOA treatment promoted Airn expression in advance, followed by regulation of Igf2r expression.

\section{Post-implantation development following artificial oocyte activation treatment}

The post-implantation development of embryos obtained from the three groups were presented in table S5. The newborn rates per pregnant recipient were similar. The birth weight of the newborn mice derived from AOA treatment embryos was significantly lower when compared with the newborn mice from ICSI derived embryos. The pups born following AOA treatment did not show any other abnormalities during early development. Moreover, the weight in all study groups was measured weekly up to 8 weeks of age (Figure S3). No significant differences were found in male or female pups among all study groups. In addition, all the weaned mice grew to adulthood. At 8 weeks of age, the offspring were mated to fertile WT mice and the mice born following AOA treatment (both female and male) mated successfully with healthy offspring born.

\section{Discussion}

Infertile couples with oocyte activation deficiency may experience ICSI failure. ICSI plus AOA has been proven to be an effective method to mitigate fertilization failure problems. However, little is known whether AOA can alter gene expression in human pre- and post-implantation embryonic development. Due to the current ethical constraints, as well as the scarcity of clinical samples, and thus clinical experiments are too difficult to carry out. The use of animal model would help to study the safety of oocyte activation, avoiding the effect of parental circumstances and genetic backgrounds. In our previous study, the oocyte activation-deficient mouse spermatozoa was identified [14]. With the release of the sperm-associated oocyte-activating factors from disrupted membranes, spermatozoa with different oocyte activation-deficient were established. This spermatozoa model could be used to study fertilization mechanisms, and to identify novel oocyte-activation strategies.
In our study, $66.67 \%$ oocytes showed no calcium rises after injection of activation-deficient sperm. Ionomycin is a commonly used assisted activator in some clinics [19], we used different concentrations of ionomycin to explore its optimal intensity. Frequency (F) reflected the total number of $\mathrm{Ca} 2+$ spikes per recording period. The calcium oscillations begin a few minutes after gamete fusion [20], occur at various frequencies and cease at the time of pronucleus (PN) formation, i.e. 4-6 $\mathrm{h}$ later [21, 22]. The frequency analysis demonstrated that the frequency of calcium oscillation will increase synchronously with the increase of AOA intensity and the distribution of frequency patterns in $2.5 \mu \mathrm{M}$ ionomycin was comparable to that in ICSI group. This means that activated deficiency of sperm could be rescued by appropriate AOA treatment. At present, a wide variety of patients with various causes of infertility may be employed by AOA treatment, which was not because of the oocyte activation deficiency [23-25]. Meanwhile, for normal sperm, we also used $2.5 \mu \mathrm{M}$ ionomycin after ICSI to explore the effect of excessive activation on embryos. The analyses of calcium oscillations showed that the extra activation (2.5 $\mu \mathrm{M}$ ionomycin) did not impact the distribution of frequency patterns (Fig. 1B). To better verify the accuracy of the analysis, the duration of a single calcium oscillation was analyzed in all study groups. Duration of the every $\mathrm{Ca} 2+$ transient was measured as the time from when the first sharp change in positive slope occurs to when it returned to the same point (Figure S4A). The results of the two methods were consistent (Figure S4B).

It is known that fertilizing sperm induce oscillations in $\mathrm{Ca}^{2+}$ levels to initiate the activation of oocytes, and intracellular calcium signaling also plays an essential role in the sequential embryonic development [26]. In addition, a close correlation between cell division and calcium availability has been reported $[27,28]$, that alteration in $\mathrm{Ca}^{2+}$ signaling may be the underlying reason for defects in cell growth and cleavage. Furthermore Wong et al. showed that the application of ionophore could overcome the negative effects caused by $\mathrm{Ca}^{2+}$ deficiency, e.g. cleavage furrow regression [29]. Our previous study also proved that the incubation of sperm heads at $37^{\circ} \mathrm{C}$ led to defects in fertilization and embryo development potential, which could be rescued with AOA [14]. Moreover, Ebner et al. reported that $\mathrm{AOA}$ with $\mathrm{Ca}^{2+}$ ionophore could improve blastocyst formation rate and clinical outcomes in infertile patients with previous embryo developmental problems [24]. In Fig. 2; Table 1, the blastocyst formation rate of group $\mathrm{C}$ was significantly lower than that of group D. The results revealed that AOA treatment could improve the blastocyst formation rate of activation-deficient sperm ICSI-derived embryos in vitro, which were consistent with the previous studies. 
Previous studies indicated that the embryo is sensitive to its very early environment in ART and may have long-lasting consequences [30,31]. With the rapid development of genome-wide transcriptome analysis, an increasing number of studies have been focusing on the different gene expression following ART manipulation. Now RNA and DNA sequencing have become more useful technologies that could help to study the expression changes observed in AOA-derived embryos. For the first time, our results provide a profile of mRNA expression in early mouse embryos with AOA treatment. A total of 54 mRNAs, among which 51 were up-regulated and 3 were down-regulated, were significantly altered in both two AOA treatment groups (ICSI-AOA group \& dICSI-AOA group) compared to ICSI group. These genes are marked in yellow in the supplement table S4. As described in our result, appropriate AOA treatment did not cause a large number of gene expression changes compared with ICSI group. The noteworthy pathways during oocyte activation were inositol phosphate metabolism and phosphatidyl inositol signaling system pathway. The KEEG analyses of these two pathways were showed in supplement Figs. 5 and 6. Oocyte activation is a $\mathrm{Ca}^{2+}$-dependent process. And $\mathrm{Ca}^{2+}$ ionophores used for AOA treatment give high permeability to cell membranes allowing $\mathrm{Ca}^{2+}$ ions to penetrate through. This $\mathrm{Ca}^{2+}$ influx from the intracellular stores, particularly the ER, induce an increase of free intracytoplasmic $\mathrm{Ca}^{2+}$ in oocytes [32]. Moreover, ionomycin might exert the substantial cellular stress by altering membrane permeability, which may differ from the physiological $\mathrm{Ca}^{2+}$ oscillatory response $[33,34]$. It is possible that these different events following ICSI-AOA trigger a different wave of gene activation, especially phosphatidyl inositol signaling pathway. In addition, a higher number of up-regulated than down-regulated genes presented in AOA treatment group, suggesting that this artificial activation step stimulate some events initiated by sperm penetration.

It is known that imprinted genes play an important role in regulating the growth and development of embryo and placenta in utero, and also in the early postnatal phase. Some non-physiological embryo culture environments or manipulation in vitro may influence the epigenetic modification of imprinted genes during early embryo-genesis [35]. Some epigenetic changes in pre-implantation embryos may further affect gene expression during fetal development stage [36].Therefore, we focused the study to explore the effect of AOA on expression of imprinted genes. In the mouse, there are about 150 known imprinted genes, many of which occur in imprinted gene clusters that are regulated together. The maternally expressed $I g f 2 r, S l c 22 a 2$, and $S l c 22 a 3$ genes and the paternally expressed lncRNA
Airn were presented in one of the gene cluster on mouse chromosome 17 [37]. Imprinting of Igf2r gene is controlled by DMR2 that contains the promoter of the long non-coding (lnc) RNA Airn, whose transcript overlaps the paternal $\operatorname{Igf} 2 r$ promoter in an antisense orientation [38]. $I g f 2 r$ is initially biallelically expressed from four-cell stage to the blastocyst and does not show maternal-specific expression until implantation $[39,40]$. The expression of $I g f 2 r$ in somatic tissues is related to the DMRs on the parental chromosomes, which DMR1 includes the Igf2r promoter, and DMR2 is located in intron 2 of Igf $2 r$, encompassing the Airn promoter. DMR2 acquires its maternal methylation during oogenesis, whereas DMR1 acquires paternal methylation after post-implantation[41]. In ICSI control blastocysts, the precise ICR border in maternal allele was maintained. However, DNA methylation had spread in the 3'direction in AOA treatment blastocysts. The results indicated that DMR2 maternal allele methylation increases or spreads in pre-implantation embryos with AOA treatment, which were consistent with that occurring during gastrulation (E6.5-E7.5). This study proposed that AOA treatment may stimulates maternal allele-specific mehtylation spreads in advance at DMR2, followed by initiation of paternal Airn transcription, resulting up regulated expression of $I g f 2 r$.

The previous studies proved that reduction of $I g f 2 r$ gene expression has functional consequences for increased birth weight [42] and the biallelic Igf2r expression $(+/$ $\mathrm{R} 2 \Delta$ ), compared with wild-type mice that have imprinted maternal-specific expression, has the decreased weight of mice [43]. Post-implantation development results indicated that the lower birth weight observed in neonates of AOA groups compared with that of ICSI group, which may be the result of IGF2R overexpression in advance. Nevertheless, no significant differences were found in post-natal growth of the pups in present study.

\section{Conclusions}

The aim of this study was to determine the effect of AOA treatment on the gene expression in the developing mouse embryo. In this study, we provide a profile of the changes in the global patterns of gene expression in ICSIAOA treatment versus ICSI generated mouse blastocysts. Another key observation in this study is that AOA treatment affects imprinted gene Igf $2 r$ expression, which is regulated by the imprinted Airn lncRNA. It is hoped that the study could provide valuable insights of AOA technology in assisted reproduction biology. And further studies are needed to evaluate the long-term effect of AOA on the offsprings. 


\section{Supplementary Information}

The online version contains supplementary material available at https://doi. org/10.1186/s12958-021-00845-7.

Additional file 1: Figure S1. Dynamic regulation of Igf2r/Airn in the preimplantation embryo. What was previously known? In preimplantation embryos or undifferentiated ES cells, lgf2r is expressed biallelically (dashed arrow) and lgf2r promoter is unmethylated on both parental alleles (white oval). Airn is silent, and promoter is methylated on the maternal allele (black hexagon) and unmethylated on the paternal allele (white hexagon). In late post-implantation or late differentiated ES cells, Igf2r is up-regulated on the maternal allele (thick green arrow), but its up-regulation is blocked on the paternal allele by Airn transcriptional interference in cis. The paternal Igf2r promoter gains DNA methylation in late post-implantation or late differentiation (black oval). Airn (wavy line) is transcribed from the unmethylated paternal allele. References: 1 (Szabo and Mann,1995), 2(Lerchner and Barlow, 1997), 3(Wang et al., 1994), 4(Stoger et al., 1993), 5(Braidotti et al., 2004), 6(Mikkelsen et al.,2007), 7(Latos, P.A.,2009), 8(Sleutels et al., 2002), 9(Latos, P.A., et al.2012), 10(Marcho, C., et al. 2015).

Additional file 2: Figure S2. Airn DMR2 MS-methylation in two flanks of $3^{\prime}$ boundary. (A) DMR2 MS-methylation state in the left side of $3^{\prime}$ boundary in mouse blastocysts. The analyzed region contains $10 \mathrm{CpG}$ sites and five clones were sequenced for each array of the group. (B) DMR2 MS-methylation state in the right side of $3^{\prime}$ boundary in mouse blastocysts. The analyzed region contains $7 \mathrm{CpG}$ sites and five clones were sequenced for each array of the group.

Additional file 3. Postnatal growth of the pups. Postnatal growth of male (A) and female (B) pups during the first 8 weeks after birth obtained following ICSI (green), ICSI-AOA (red), or dICSI-AOA (blue). Data are Mean $\pm S D$. X-axis: time in weeks; $Y$-axis: body weight in $\mathrm{g}$.

Additional file 4: Figure S4. Duration of the every $\mathrm{Ca} 2+$ transient. (A) Schematic showing how duration times were measured. (B) Graph comparing the mean duration times of a single $\mathrm{Ca} 2+$ transients in all groups. Asterisks indicate significant differences compared to ICSI control group.

Additional file 5: Figure S5. lositol phosphate metabolism by The Kyoto Encyclopedia of Genes and Genomes (KEGG) analysis.

Additional file 6: Figure S6. Posphatidylinositol signaling system by The Kyoto Encyclopedia of Genes and Genomes (KEGG) analysis.

Additional file 7.

\section{Acknowledgements}

We gratefully acknowledge the staff of the Department of Assisted Reproduction in Shanghai Ninth People's Hospital for their contribution.

\section{Authors' contributions}

Mingru Yin and Qifeng Lyu developed the experimental designs. Mingru Yin, Pengcheng Kong and Weina Yu conducted the experiments and wrote the manuscript. Qifeng Lyu and Qianqian Zhu revised the manuscript and data interpretation. Wenzhi Li and Hui Long participated in critical discussions and data analysis. The author(s) read and approved the final manuscript.

\section{Funding}

This work was supported by the National Nature Science Foundation of China (grant nos. 81701523, 81871163, 81801538, 81903324) and National Key Research and Development Program of China (Grant number 2018YFC1003001).

\section{Availability of data and materials}

All data generated through this study are included in this article.

\section{Declarations}

Ethics approval and consent to participate

All procedures were approved by the Ethic Committee of Shanghai Ninth People's Hospital affiliated to shanghai Jiao Tong University, School of Medicine (Approval No.: HKDL [2017]155).

\section{Consent for publication}

Not applicable.

\section{Competing interests}

The authors declare that they have no competing interests.

Received: 20 July 2021 Accepted: 7 October 2021

Published online: 04 November 2021

\section{References}

1. Palermo GD, O'Neill CL, Chow S, Cheung S, Parrella A, Pereira N, Rosenwaks Z. Intracytoplasmic sperm injection: state of the art in humans. Reproduction. 2017;154:F93-110. https://doi.org/10.1530/REP-17-0374.

2. Esfandiari N, Javed MH, Gotlieb L, Casper RF. Complete failed fertilization after intracytoplasmic sperm injection--analysis of 10 years' data. Int J Fertil Womens Med. 2005;50:187-92.

3. Machaty Z, Miller AR, Zhang L. Egg Activation at Fertilization. Adv Exp Med Biol. 2017;953:1-47. https://doi.org/10.1007/978-3-319-46095-6_1.

4. Machaty Z. Signal transduction in mammalian oocytes during fertilization. Cell Tissue Res. 2016;363:169-83. https://doi.org/10.1007/ s00441-015-2291-8.

5. Yeste M, Jones C, Amdani SN, Coward K. Oocyte Activation and Fertilisation: Crucial Contributors from the Sperm and Oocyte. Results Probl Cell Differ. 2017;59:213-39. https://doi.org/10.1007/978-3-319-44820-6_8.

6. Kashir J, Heindryckx B, Jones C, De Sutter P, Parrington J, Coward K. Oocyte activation, phospholipase C zeta and human infertility. Hum Reprod Update. 2010;16:690-703. https://doi.org/10.1093/humupd/ dmq018.

7. Davies MJ, Moore VM, Willson KJ, Van Essen P, Priest K, Scott H, Haan EA, Chan A. Reproductive technologies and the risk of birth defects. N Engl J Med. 2012;366:1803-13. https://doi.org/10.1056/NEJMoa1008095.

8. Li B, Zhou Y, Yan Z, Li M, Xue S, Cai R, Fu Y, Hong Q, Long H, Yin M, et al. Pregnancy and neonatal outcomes of artificial oocyte activation in patients undergoing frozen-thawed embryo transfer: a 6-year population-based retrospective study. Arch Gynecol Obstet. 2019;300:1083-92. https://doi.org/10.1007/s00404-019-05298-3.

9. Mateizel I, Verheyen G, Van de Velde H, Tournaye H, Belva F. Obstetric and neonatal outcome following ICSI with assisted oocyte activation by calcium ionophore treatment. J Assist Reprod Genet. 2018;35:1005-10. https://doi.org/10.1007/s10815-018-1124-6.

10. Vanden Meerschaut F, Nikiforaki D, De Roo C, Lierman S, Qian C, SchmittJohn T, De Sutter P, Heindryckx B. Comparison of pre- and post-implantation development following the application of three artificial activating stimuli in a mouse model with round-headed sperm cells deficient for oocyte activation. Hum Reprod. 2013;28:1 190-8. https://doi.org/10.1093/ humrep/det038.

11. Nomikos M, Swann K, Lai FA. Starting a new life: sperm PLC-zeta mobilizes the $\mathrm{Ca} 2+$ signal that induces egg activation and embryo development: an essential phospholipase $\mathrm{C}$ with implications for male infertility. Bioessays. 2012;34:126-34. https://doi.org/10.1002/bies.201100127.

12. Nakai M, Ito J, Sato K, Noguchi J, Kaneko H, Kashiwazaki N, Kikuchi K. Pre-treatment of sperm reduces success of ICSI in the pig. Reproduction. 2011;142:285-93. https://doi.org/10.1530/REP-11-0073.

13. Anzalone DA, luso D, Czernik M, Ptak G, Loi P. Plasma membrane and acrosome loss before ICSI is required for sheep embryonic development. J Assist Reprod Genet. 2016;33:757-63. https://doi.org/10.1007/ s10815-016-0709-1.

14. Long H, Lu SS, Kuang YP, Yan ZG, Liang HX, Yu S, Chai WR, Yan Z, Lyu QF. Incubation of sperm heads impairs fertilization and early embryo development following intracytoplasmic sperm injection (ICSI) by decreasing 
oocyte activation in mice. Biotechnol Lett. 2013;35:1823-9. https://doi. org/10.1007/s10529-013-1287-2.

15. Kong P, Yin M, Chen D, Li S, Li Y, Xing F, Jiang M, Fang Z, Lyu Q, Chen X. Effects of the histone deacetylase inhibitor 'Scriptaid' on the developmental competence of mouse embryos generated through round spermatid injection. Hum Reprod. 2017;32:76-87. https://doi.org/10. 1093/humrep/dew290.

16. Yoon SY, Jellerette T, Salicioni AM, Lee HC, Yoo MS, Coward K, Parrington J, Grow D, Cibelli JB, Visconti PE, et al. Human sperm devoid of PLC, zeta 1 fail to induce $\mathrm{Ca}(2+)$ release and are unable to initiate the first step of embryo development. J Clin Invest. 2008;1 18:3671-81. https://doi.org/10 $1172 / \mathrm{JCl} 36942$.

17. Vanden Meerschaut F, Leybaert L, Nikiforaki D, Qian C, Heindryckx B, De Sutter P. Diagnostic and prognostic value of calcium oscillatory pattern analysis for patients with ICSI fertilization failure. Hum Reprod. 2013;28:87-98. https://doi.org/10.1093/humrep/des368.

18. Lyu QF, Deng L, Xue SG, Cao SF, Liu XY, Jin W, Wu LQ, Kuang YP. New technique for mouse oocyte injection via a modified holding pipette. Reprod Biomed Online. 2010;21:663-6. https://doi.org/10.1016/j.rbmo. 2010.07.004.

19. Vanden Meerschaut F, Nikiforaki D, Heindryckx B, De Sutter P. Assisted oocyte activation following ICSI fertilization failure. Reprod Biomed Online. 2014;28:560-71. https://doi.org/10.1016/j.rbmo.2014.01.008.

20. Deguchi R, Shirakawa H, Oda S, Mohri T, Miyazaki S. Spatiotemporal analysis of $\mathrm{Ca}(2+)$ waves in relation to the sperm entry site and animalvegetal axis during $\mathrm{Ca}(2+)$ oscillations in fertilized mouse eggs. Dev Biol. 2000;218:299-313. https://doi.org/10.1006/dbio.1999.9573.

21. Jellerette T, He CL, Wu H, Parys JB, Fissore RA. Down-regulation of the inositol 1,4,5-trisphosphate receptor in mouse eggs following fertilization or parthenogenetic activation. Dev Biol. 2000;223:238-50. https://doi.org/ 10.1006/dbio.2000.9675.

22. Marangos P, FitzHarris G, Carroll J. Ca2+ oscillations at fertilization in mammals are regulated by the formation of pronuclei. Development. 2003;130:1461-72. https://doi.org/10.1242/dev.00340.

23. Karabulut S, Aksunger O, Ata C, Sagiroglu Y, Keskin I. Artificial oocyte activation with calcium ionophore for frozen sperm cycles. Syst Biol Reprod Med. 2018;64:381-8. https://doi.org/10.1080/19396368.2018.1452311.

24. Ebner T, Oppelt P, Wober M, Staples P, Mayer RB, Sonnleitner U, BulfonVogl S, Gruber I, Haid AE, Shebl O. Treatment with Ca2+ ionophore improves embryo development and outcome in cases with previous developmental problems: a prospective multicenter study. Hum Reprod. 2015:30:97-102. https://doi.org/10.1093/humrep/deu285.

25. Lv M, Zhang D, He X, Chen B, Li Q, Ding D, Hao Y, Xue R, Ji D, Zou W, et al. Artificial oocyte activation to improve reproductive outcomes in couples with various causes of infertility: a retrospective cohort study. Reprod Biomed Online. 2020;40:501-9. https://doi.org/10.1016/j.rbmo. 2020.01.001.

26. Ducibella T, Fissore R. The roles of $\mathrm{Ca} 2+$, downstream protein kinases, and oscillatory signaling in regulating fertilization and the activation of development. Dev Biol. 2008;315:257-79. https://doi.org/10.1016/j.ydbio. 2007.12.012.

27. Berridge MJ, Bootman MD, Lipp P. Calcium--a life and death signal. Nature. 1998:395:645-8. https://doi.org/10.1038/27094.

28. Berridge MJ. Calcium signalling and cell proliferation. Bioessays. 1995;17:491-500. https://doi.org/10.1002/bies.950170605.

29. Wong R, Hadjiyanni I, Wei HC, Polevoy G, McBride R, Sem KP, Brill JA. PIP2 hydrolysis and calcium release are required for cytokinesis in Drosophila spermatocytes. Curr Biol. 2005;15:1401-6. https://doi.org/10.1016/j.cub. 2005.06.060.
30. Kleijkers SH, van Montfoort AP, Smits L, Viechtbauer W, Roseboom TJ, Nelissen EC, Coonen E, Derhaag JG, Bastings L, Schreurs IE, et al. IVF culture medium affects post-natal weight in humans during the first 2 years of life. Hum Reprod. 2014;29:661-9. https://doi.org/10.1093/humrep/ deu025.

31. Fernandez-Gonzalez R, Moreira P, Bilbao A, Jimenez A, Perez-Crespo M, Ramirez MA, Rodriguez De Fonseca F, Pintado B, Gutierrez-Adan A. Long-term effect of in vitro culture of mouse embryos with serum on mRNA expression of imprinting genes, development, and behavior. Proc Natl Acad Sci U S A. 2004;101:5880-5. https://doi.org/10.1073/pnas.03085 60101.

32. Vasilev F, Chun JT, Gragnaniello G, Garante E, Santella L. Effects of ionomycin on egg activation and early development in starfish. PLoS One. 2012;7:e39231. https://doi.org/10.1371/journal.pone.0039231.

33. Santella L, Dale B. Assisted yes, but where do we draw the line? Reprod Biomed Online. 2015;31:476-8. https://doi.org/10.1016/j. rbmo.2015.06.013.

34. van Blerkom J, Cohen J, Johnson M. A plea for caution and more research in the 'experimental' use of ionophores in ICSI. Reprod Biomed Online. 2015;30:323-4. https://doi.org/10.1016/j.rbmo.2015.02.002.

35. Young LE, Fairburn HR. Improving the safety of embryo technologies: possible role of genomic imprinting. Theriogenology. 2000;53:627-48. https://doi.org/10.1016/s0093-691x(99)00263-0.

36. Dean W, Bowden L, Aitchison A, Klose J, Moore T, Meneses JJ, Reik W, Feil R. Altered imprinted gene methylation and expression in completely ES cell-derived mouse fetuses: association with aberrant phenotypes. Development. 1998;125:2273-82.

37. Zwart R, Sleutels F, Wutz A, Schinkel AH, Barlow DP. Bidirectional action of the lgf2r imprint control element on upstream and downstream imprinted genes. Genes Dev. 2001;15:2361-6. https://doi.org/10.1101/ gad.206201.

38. Santoro F, Mayer D, Klement RM, Warczok KE, Stukalov A, Barlow DP, Pauler FM. Imprinted Igf2r silencing depends on continuous Airn IncRNA expression and is not restricted to a developmental window. Development. 2013;140:1184-95. https://doi.org/10.1242/dev.088849.

39. Szabo PE, Mann JR. Allele-specific expression and total expression levels of imprinted genes during early mouse development: implications for imprinting mechanisms. Genes Dev. 1995;9:3097-108. https://doi.org/10. 1101/gad.9.24.3097.

40. Lerchner W, Barlow DP. Paternal repression of the imprinted mouse Igf2r locus occurs during implantation and is stable in all tissues of the postimplantation mouse embryo. Mech Dev. 1997;61:141-9. https://doi.org/ 10.1016/s0925-4773(96)00630-2.

41. Stoger R, Kubicka P, Liu CG, Kafri T, Razin A, Cedar H, Barlow DP. Maternalspecific methylation of the imprinted mouse lgf2r locus identifies the expressed locus as carrying the imprinting signal. Cell. 1993;73:61-71. https://doi.org/10.1016/0092-8674(93)90160-r.

42. Young LE, Fernandes K, McEvoy TG, Butterwith SC, Gutierrez CG, Carolan C, Broadbent PJ, Robinson JJ, Wilmut I, Sinclair KD. Epigenetic change in IGF2R is associated with fetal overgrowth after sheep embryo culture. Nat Genet. 2001;27:153-4. https://doi.org/10.1038/84769.

43. Wutz A, TheussI HC, Dausman J, Jaenisch R, Barlow DP, Wagner EF. Non-imprinted lgf2r expression decreases growth and rescues the Tme mutation in mice. Development. 2001;128:1881-7.

\section{Publisher's Note}

Springer Nature remains neutral with regard to jurisdictional claims in published maps and institutional affiliations. 